\title{
Baryonium, a common ground for atomic and high energy physics
}

\author{
S. Wycech · J.-P. Dedonder $\cdot$ B. Loiseau
}

Published online: 3 April 2015

(C) The Author(s) 2015. This article is published with open access at Springerlink.com

\begin{abstract}
Indications of the existence of quasi-bound states in the $N \bar{N}$ system are presented. In their measurements, the BES collaboration has discoverd a broad enhancement close to the $p \bar{p}$ threshold in the $S$ wave, isospin 0 state formed in radiative decays of $J / \psi$. Another enhancement located about $50 \mathrm{MeV}$ below the threshold was found in mesonic decays of $J / \psi$. In terms of the Paris potential model it was shown that these are likely to represent the same state. Antiprotonic atomic data provide some support for this interpretation and indicate the existence of another fairly narrow quasi-bound state in a $P$ wave.
\end{abstract}

Keywords Baryonium $\cdot \mathrm{J} /$ psi decays $\cdot$ antiprotonic atoms $J / \psi$

\section{Introduction}

Nucleon-antinucleon quasi-bound states, or states coupled to these, were searched for in the days of LEAR at CERN. Nothing has been found, but broad states or states close to the threshold were not excluded. References [1], [2] indicate conclusions of a long series of measurements. In reference [1] a search for narrow signals in the $\gamma$ spectrum from $p \bar{p}$

Proceedings of the International Conference on Exotic Atoms and Related Topics (EXA 2014), Vienna, Austria, 15-19 September

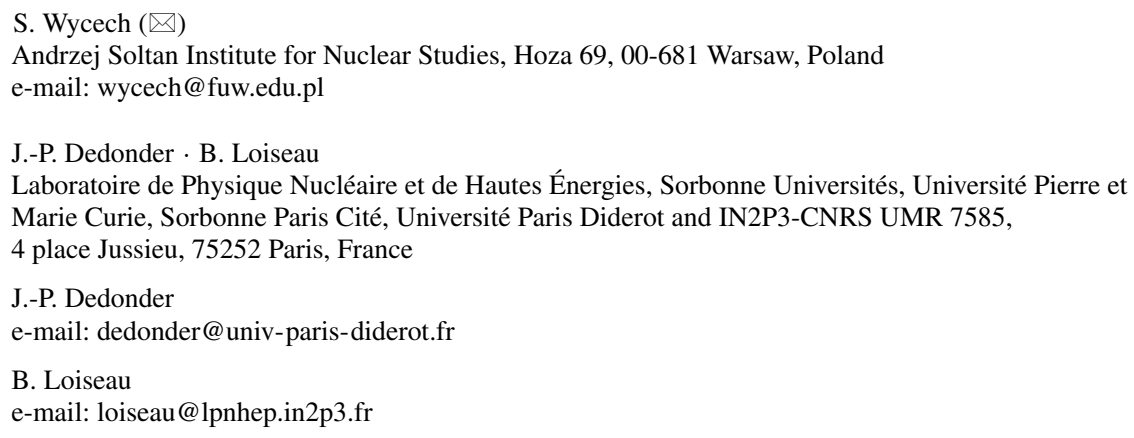


Table 1 Low energy $p \bar{p}$ states allowed in the $J / \psi \rightarrow \gamma p \bar{p}$ decays. The first column gives decay modes and specifies the internal states of $p \bar{p}$ pair. For both photon and $J / \psi$ the $J^{P C}=1^{(--)}$. The second column gives $J^{P C}$ for the $p \bar{p}$ system

\begin{tabular}{ll}
\hline Decay mode & $J^{P C}(p \bar{p})$ \\
\hline$\gamma p \bar{p}\left({ }^{1} S_{0}\right)$ & $0^{-+}$ \\
$\gamma p \bar{p}\left({ }^{3} P_{0}\right)$ & $0^{++}$ \\
$\gamma p \bar{p}\left({ }^{3} P_{1}\right)$ & $1^{++}$ \\
\hline
\end{tabular}

Table 2 Decay modes and the $p \bar{p}$ states allowed in the $J / \psi \rightarrow$ boson $p \bar{p}$ decays

\begin{tabular}{lll}
\hline Decay mode & Branching & $p \bar{p}$ states allowed \\
\hline$\gamma p \bar{p}$ & $3.8( \pm 1.0) \cdot 10^{-4}[8]$ & ${ }^{1} S_{0},{ }^{3} P_{1},{ }^{3} P_{0}$ \\
$\omega p \bar{p}$ & $1.1( \pm 0.15) \cdot 10^{-3}[9]$ & ${ }^{11} S_{0},{ }^{13} P_{1},{ }^{13} P_{0}$ \\
$\pi^{0} p \bar{p}$ & $1.19( \pm .08) \cdot 10^{-3}[8]$ & ${ }^{33} S_{1},{ }^{31} P_{1}$ \\
$p \bar{p}$ & $2.12( \pm 0.1) \cdot 10^{-3}[8]$ & ${ }^{13} S_{1}$ \\
\hline
\end{tabular}

annihilation at rest was performed and no discoveries were found in the region below 1770 $\mathrm{MeV}$ and $\Gamma<25 \mathrm{MeV}$. Another experiment looking for missing mass in the reactions $p \bar{p} \rightarrow \pi^{-} X$ and $\bar{p} d \rightarrow p X$ brought similar conclusions. On the experimental side, one possible reason for the failure is the large background due to annihilation processes. Another is the large number of allowed partial waves. On the theory side, it was assumed that the annihilation reaction involve $\sim 2 M_{p}$ mass transfer and by the uncertainty principle it has to be very short ranged. It was thus expected that widths of quasi-bound states might be narrow. The first part of the argument is still true but it is also known from scattering data that the annihilation potential is strong already at $p-\bar{p}$ separation of $1 \mathrm{fm}$.

A convincing detection requires selective experiments, and the first such measurement is that of the decay

$$
J / \psi \rightarrow \gamma p \bar{p},
$$

studied by the BES Collaboration [3]. A strong threshold enhancement appears in the invariant $p \bar{p}$ mass distribution (see Fig. 3). There are three final $p \bar{p}$ states allowed by $P$ and $C$ conservation in the $\gamma p \bar{p}$ channel. These are listed in Tables 1 and 2 and denoted by ${ }^{2 S+1} L_{J}$ or ${ }^{2 I+1,2 S+1} L_{J}, S, L, J$ being the spin, angular momentum, total momentum of the pair and $I$ denotes the isospin. Radiative decay does not conserve isospin but already in ref. [3] and ref. [4] it was realized that $I=0$ is the state which leads to the enhancement. From potential descriptions of $N \bar{N}$ interactions based on the $G$-parity rule it is known that the pion exchange potential is very strong in this state, being capable to form bound states. On the other hand the annihilation and short range interactions act repulsively, thus quasibound states are not guaranteed. In particular the Paris potential generates a $52 \mathrm{MeV}$ broad quasi-bound state at $4.8 \mathrm{MeV}$ below threshold [5] but the the Bonn-Jülich potential does not generate bound state in this wave [6]. Both can describe the threshold enhancement [6],[7].

The radiative process itself is puzzling as the decay rate is comparable to the mesonic decay rates although the conventional coupling constants $\alpha / g_{N N \text {, meson }}^{2}$ are $\sim 10^{-3}$. Thus, a strong enhancement mechanism has to exist. In Section 2, this question is discussed jointly with the origin of the threshold enhancement. 
Fig. 1 The photon is emitted either from $J / \psi$ or during the hadronisation stage of the process and the final baryons are formed in the $S$ wave

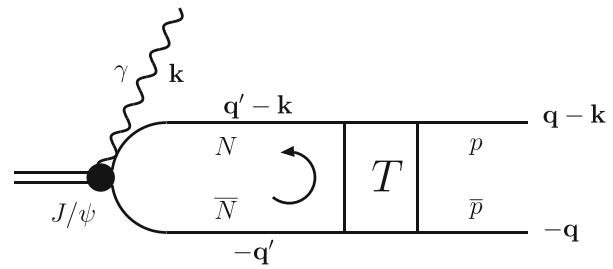

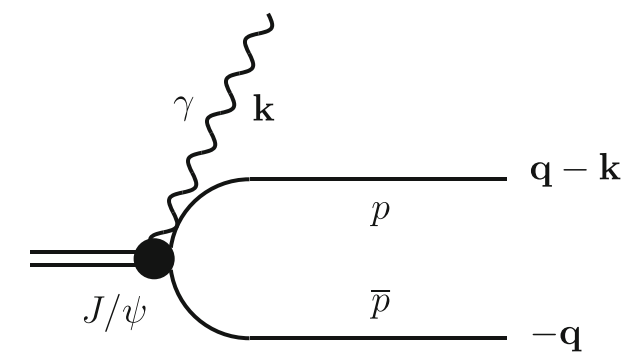

Fig. 2 Final state interactions described by half-off shell $T$ matrix generated by the Paris potential
There are other indications of $N \bar{p}$ structures existing below threshold coming from antiprotonic atoms. These are discussed in Section 3. In Section 4 we list possible experimental researches of the baryonia which could be performed in a near future.

\section{Final state interactions in $J / \psi$ decays}

We have attempted calculations of the radiative and mesonic decay rates presented in Table 2, assuming that mesons are emitted in the final states of the decay when the baryons have been formed. With conventional meson-nucleon coupling constants this model reproduces the branching ratios of $p \bar{p}-$ meson channels relative to the basic $p \bar{p}$ channel [10]. It offers also a consistent description of the spectra in cases of $\pi^{0}$ and $\omega$ meson production at the expense of one free parameter, the $N \bar{N}$ formation radius $R=0.28 \mathrm{fm}$. However, this model fails in the description of radiative decays in two ways: first, the branching ratio is only about $1 / 3$ of the experimental one, and second, the threshold enhancement is not reproduced. The transition to ${ }^{1} S_{0}$ state is a magnetic one and it turns out that in the intermediate stage of such decay one has both $p \bar{p}$ and $n \bar{n}$ as intermediate states. Since magnetic moments of $p$ and $n$ have opposite sign the effect of final state enhancement cancels strongly. At the same time such a model indicates that final state interactions increase the overall decay rate by an order of magnitude in the $I=1$ states.

In this note we report an extension of the final state interactions (FSI) calculations of ref. [7] which is now used to cover the whole photon spectrum. The basic assumption of this approach (also that in ref. [6]) is that the photon is emitted before the baryons are formed. The two related processes are plotted in diagrams Figs. 1, 2 and the FSI is calculated in terms of half-off shell $T$ matrix generated by the Paris potential [5] plotted in Fig. 4. This approach allows to calculate the spectrum but not the absolute decay rate. One free parameter, the radius $R(=0.28 \mathrm{fm})$ of a Gaussian source function is used to describe the creation of a $\gamma p \bar{p}$ state (see Fig. 1). However, in order to reproduce in a better way both maxima in Fig. 3 (the $X(1859)$ and $X(2170)$ in BES terminology), it turns out profitable to assume the radius to be weakly dependent on the photon 


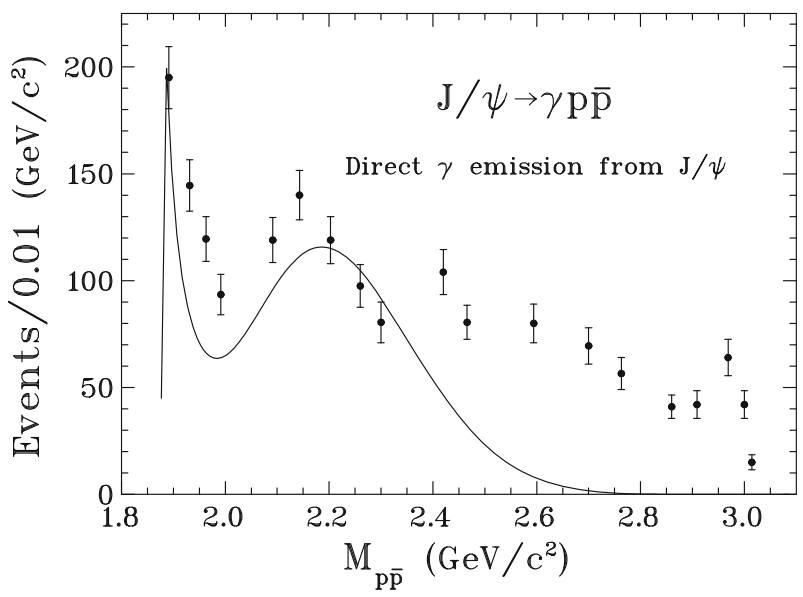

Fig. 3 The $p \bar{p}$ invariant mass obtained under the assumption that photon is emitted before the baryons are formed. The missing strength at large $p \bar{p}$ invariant mass, $M_{p \bar{p}}$, comes from the photon radiated by final hadrons [10]. The experimental points from ref. [3]

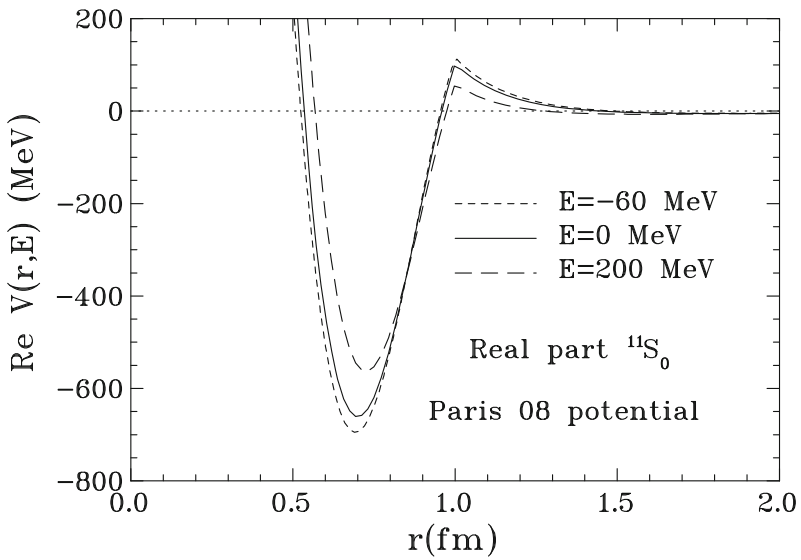

Fig. 4 The Paris $N \bar{N}$ real potential in the ${ }^{11} S_{0}$ wave. It generates a $50 \mathrm{MeV}$ broad quasi-bound state at $\sim$ $5 \mathrm{MeV}$ binding. The well and barrier structure generate the shape resonance visible in the spectrum in Fig. 3 at $2170 \mathrm{MeV}$

energy. It was found to change from $0.28 \mathrm{fm}$ at maximal $k \sim 1.2 \mathrm{GeV}$ to $0.39 \mathrm{fm}$ at $k=0$ (Fig. 4).

Inspection of Fig. 3 shows that both states may be reproduced by the potential $N \bar{N}$ interactions related at large distances via G-parity transformation to the $N N$ interactions. However, the proper description of both peaks involves a distant extrapolation of the $T_{N} \bar{N}$ matrices off energy shell, which corresponds to very short ranged interactions. This figure shows also that a sizable portion of the spectrum is missing and this part comes from the photon emission by the $N \bar{N}$ pair and exchange currents [10].

As already discussed, the threshold enhancement indicates a "nearby singularity" that might describe an analogue of the bound state or the virtual state known from the physics 


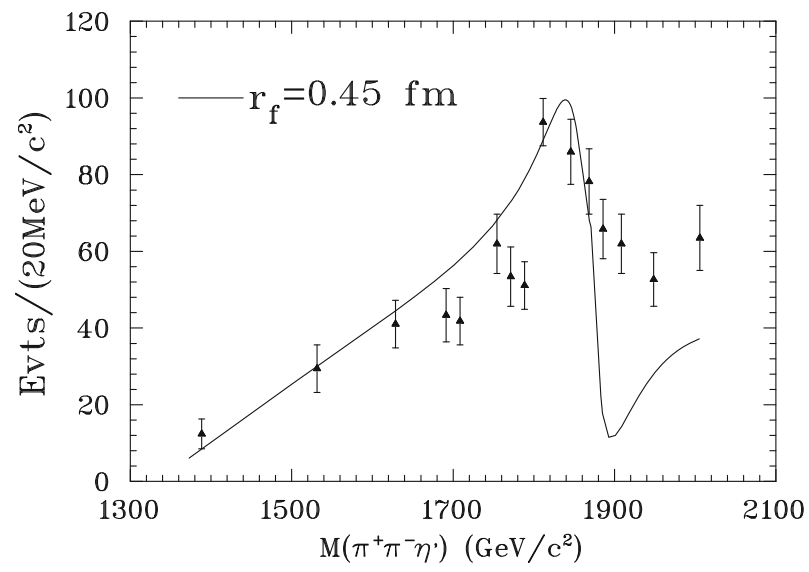

Fig. 5 The spectral function $\mathrm{X}_{S}$ representing the $X(1835)$ shape. The parameter of the annihilation range is $r_{f}=0.45 \mathrm{fm}$. This $S$-wave contribution has been normalized to reproduce the data close to the $X(1835)$ peak. The experimental points are from ref. [11], calculation from ref. [12]

Table 3 Ratios of $N(n \bar{p})$ and $N(p \bar{p})$ capture rates from atomic states

\begin{tabular}{llll}
\hline Atom & Lower & Upper & Radiochemistry \\
\hline${ }^{96} \mathrm{Zr}$ & $0.95(9)$ & $1.53(29)$ & $2.6(3)$ \\
${ }^{124} \mathrm{Sn}$ & $1.79(10)$ & $2.44(39)$ & $5.0(6)$ \\
${ }^{106} \mathrm{Cd}$ & $1.64(80)$ & $2.10(80)$ & $0.5(1)$ \\
${ }^{112} \mathrm{Sn}$ & $1.90(13)$ & $2.43(49)$ & $0.79(14)$ \\
\hline
\end{tabular}

The last column shows experimental numbers from radiochemical experiments. Other columns (see text) give ratios calculated with optical potential and plausible nuclear densities based on experimental results from Ref. [13].

of two nucleons. To discern these possibilities one has to test directly the sub-threshold region.

\section{Studies of the $N \bar{N}$ sub-threshold region}

One way to look below the threshold is the detection of $N \bar{N}$ decay products. The specific decay mode

$$
J / \psi \rightarrow \gamma \pi^{+} \pi^{-} \eta^{\prime}
$$

has been studied by the BES collaboration [11]. This reaction is attributed by BES to an intermediate $p \bar{p}$ configuration in the $J^{P C}(p \bar{p})=0^{-+}$state, that is in the ${ }^{1} S_{0}$ wave. A peak in the invariant mass of the mesons has been observed and interpreted as a new baryon state and named X(1835).

Under the assumption that all mesons are produced in relative $S$ waves, the reaction (2), if attributed to an intermediate $p \bar{p}$, is even more restrictive than the reaction (1). It allows only one intermediate state, the $p \bar{p}{ }^{1} S_{0}$, which coincides with the previous findings. The intermediate state of $p \bar{p}$ in reaction (2) is possible but not warranted. In ref. [12] a more consistent interpretation is obtained with the dominance of the ${ }^{11} S_{0}$ state which is a mixture 
Table 4 Binding energies in $\mathrm{MeV}$ of the close to threshold quasi-bound states in the Paris potential [5]

\begin{tabular}{ll}
\hline${ }^{2 T+1}{ }^{2 S+1} L_{J}$ & $E-i \Gamma / 2$ \\
\hline${ }^{11} S_{0}$ & $-4.8-i 26$ \\
${ }^{33} P_{1}$ & $-4.5-i 9.0$ \\
\hline
\end{tabular}

of $p \bar{p}$ and $n \bar{n}$ pairs. It has been argued that the peak is due to an interference of a quasibound, isospin $0, N \bar{N}$ state with a background amplitude. A typical interference pattern obtained in this way is plotted in Fig. 5. It is fairly close to the data. The same quasi-bound state was found in ref. [7] to be responsible for the threshold enhancement in reaction (1). In this sense the Paris potential unifies the two effects and attributes it to single quasi-bound state with an energy dependent width.

Testing the subthreshold amplitudes may be also realized in few body systems, in particular in light antiprotonic atoms or at extreme nuclear peripheries. In these conditions nucleons are bound and the effective subthreshold energies are composed of binding energies and recoil of the $N \bar{p}$ pair with respect to the rest of the system. For valence nucleons the $E_{\text {binding }}+E_{\text {recoil }}$ may reach down to $-40 \mathrm{MeV}$ below threshold. Let us indicate an atomic experiment that discovered an interesting anomaly. Table 3 shows the ratios of antiproton capture rates on neutrons and protons $C(n \bar{p}) / C(p \bar{p})$ bound to nuclear peripheries. These reflect the ratios of neutron and proton densities. The second and third columns indicate such ratios extracted from the widths of two antiprotonic atomic levels the "lower" and the "upper" one. These widths are determined at nuclear densities $\sim 10 \%$ and $\sim 5 \%$ of the central density $\rho_{0}$. The last column is obtained with radiochemical studies of final nuclei with one neutron or one proton removed in the annihilation reaction [13]. The latter process is localized at densities $\rho \sim 10^{-3} \rho_{0}$. In standard nuclei shown in the upper part of the table the ratios $N(n \bar{p}) / N(p \bar{p}) \sim \rho_{n} / \rho_{p}$ increase at nuclear peripheries. However, in some nuclei characterized by small protonbinding, indicated in the lower part of the table, and typical ( $\sim 8 \mathrm{MeV}$ ) neutron binding the experimental ratio $N(n \bar{p}) / N(p \bar{p})$ suddenly drops at extreme nuclear peripheries. That effect cannot be explained by the nuclear structure alone and we attribute it to the existence of a narrow bound state in the $N \bar{N}$ system. Such a narrow state is in fact predicted by the Paris potential in the ${ }^{33} P_{1}$ wave, see Table 4.

A similar effect is indicated by studies of experimental absorption lengths in light antiprotonic atoms [14], [15]. From the lower and upper atomic level widths one can extract average $S$-wave absorption lengths $\operatorname{Im} a$ and $P$-wave absorption volumes $\operatorname{Im} b$. The results shown in Fig. 6 indicate increase of the absorption in the $S$ wave down below the threshold. This result is consistent with the presence of the $X(1855)$ state. The absorption volume extracted from antiprotonic deuterium indicates some enhancement possibly related to radiochemical anomalies observed in nuclei with loosely bound protons and interpreted in terms of the Paris potential as the $P$-wave bound state.

\section{New era}

Following the indications from antiprotonic atoms and from BES experiments the baryonia should be searched in the region of $0-60 \mathrm{MeV}$ below the $N \bar{N}$ threshold. With the new antiprotonic beams expected to operate in J-PARC and FAIR it would be advisable to repeat two old experiments possibly at different energies, possibly with polarized particles. 


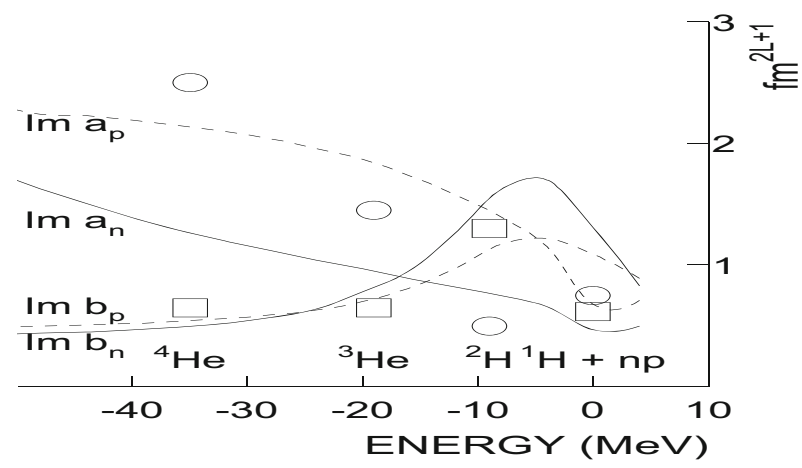

Fig. 6 The absorptive parts of spin-isospin averaged $N \bar{p}$ scattering parameters extracted from the atomic level widths in $\mathrm{H},{ }^{2} \mathrm{H},{ }^{3} \mathrm{He}$ and ${ }^{4} \mathrm{He} \bar{p}$. Rectangles: $S$ waves and ellipses : $P$ waves. The bottom scale indicates the energy below threshold. The curves, calculated with the Paris potential, give the amplitudes separately: $a_{n(p)}$ denote the $n \bar{p}$ or $p \bar{p} S$-wave scattering lengths. Similarly $b_{n(p)}, b_{p(p)}$ are the corresponding $P$-wave volumes. The vertical scale means $\mathrm{fm}$ for the lengths and $\mathrm{fm}^{3}$ for the volumes. The strong increase of absorption in the $\bar{p} p S$ wave is attributed mainly to the ${ }^{11} S_{0}$ state

- The search for narrow signals in the $\gamma$-spectrum from $p \bar{p}$ annihilation was performed at rest [1]. The signals (in the region that we expect them now to exist) were covered by heavy background due to $\pi^{0}$ decays and $\pi^{-} p \rightarrow \gamma n$. It would be better to perform this experiment with higher energy antiprotons (a few hundred $\mathrm{MeV} / \mathrm{c}$ ) which could shift the expected signal away from the heavy background region.

- $\quad$ The $\bar{p} d \rightarrow p X$ experiment [2] was performed at $1.3 \mathrm{GeV} / \mathrm{c}$. This gives rather small chance of $p \bar{p}$ coupling in the statistically insignificant ${ }^{11} S_{0}$ wave. Lower energies and polarized (one or two particles) would reduce the background.

New instructive experiments that possibly could be performed at FLAIR are:

- Fine structure splitting in light antiprotonic atoms ${ }^{1} \mathrm{H},{ }^{2} \mathrm{H},{ }^{3} \mathrm{H},{ }^{3} \mathrm{He},{ }^{4} \mathrm{He}$ would allow to trace energy dependenc of the selected $\bar{p} N$ amplitudes in the subthreshold region down to $\sim-40 \mathrm{MeV}$.

- Studies of mesons emitted from annihilations of $\bar{p}$ at nuclear peripheries. In particular nuclei with closed shells with one loosely bound valence nucleon could be profitable. In the latter case the baryonium signal would be separated from a complicated background due to other annihilation channels.

Acknowledgments This work has been partially supported by a grant from the French-Polish exchange program COPIN/CNRS- IN2P3, collaboration 05-115. SW was also supported by Narodowe Centrum Nauki grant 2011/03/B/ST2/00270

Open Access This article is distributed under the terms of the Creative Commons Attribution License which permits any use, distribution, and reproduction in any medium, provided the original author(s) and the source are credited.

Conflict of interests The authors declare that they have no conflict of interest. 


\section{References}

1. Adiels, L., et al.: Phys. Lett. B 182, 405 (1986)

2. Bertini, R., et al.: Nucl. Phys. B 209, 269 (1982)

3. Bai, J.Z., et al., (BES Collaboration): Phys. Rev. Lett. 91, 022001 (2003)

4. Ablikim, M., et al., (BES Collaboration): Phys. Rev. Lett. 108, 112003 (2012)

5. El-Bennich, B., Lacombe, M., Loiseau, B., Wycech, S.: Phys. Rev. C79, 054001 (2009)

6. Haidenbauer, J., Meißner, U.-G., Sibirtsev, A.: Phys. Rev. D74, 017501 (2006)

7. Loiseau, B., Wycech, S.: Phys. Rev. C72, 011001 (2005)

8. Nakamura, K. for Particle Data Group: J. Phys. G 37, 07021 (2010)

9. Ablikim, M. for BES Collaboration.: Eur. Phys. Journ. C53, 15(2008)

10. Dedonder, J.-P., Loiseau, B., Wycech, S.: to be submitted to Phys. Rev. C

11. Ablikim, M. for BES Collaboration.: Phys. Rev. Lett. 95, 262001 (2005)

12. Dedonder, J.-P., Loiseau, B., Wycech, S.: Phys. Rev. C80, 045209 (2009)

13. Lubinski, P., et al.: Phys. Rev. C 57, 2962 (1997)

14. Augsburger, M., et al.: Phys. Lett. B461, 417 (1999)

15. Schneider, M., et al.: Z. Phys. 217, A338 (1991) 